\title{
Allergic rhinitis in children
}

The authors of this Clinical Review (BMJ 2014;349:g4153, doi:10.1136/bmj.g4153) would like to clarify the ages at which fluticasone propionate and levocetirizine can be prescribed to children. Their article incorrectly noted that both fluticasone propionate and levocetirizine can only be given to children aged 6 years and above. In fact, fluticasone propionate can be used in children from the age of 4 , and levocetirizine can be used in children from the age of 2 .

Cite this as: BMJ 2014;349:94923

๑ BMJ Publishing Group Ltd 2014 